\title{
Les pratiques ostensives des enseignants d'EPS révélatrices du rapport au savoir en natation
}

\author{
*Donia Sghaier ${ }^{1,2}$, Hejer Ben Jomâa ${ }^{3,4}$, Mohamed Mami ${ }^{1,2}$, Anissa Bouassida ${ }^{1,2}$ \\ ${ }^{\prime}$ (Institut Supérieur du Sport et de l'Education Physique du Kef/ Université de Jendouba, Tunisie) \\ ${ }^{2}$ (Unité de Recherche de la Performance Sportive et de la Réabilitation/Université de Jendouba, Tunisie) \\ 3 (Institut Supérieur du Sport et de l'Education Physique de Ksar Saïd/ Université de la Manouba, Tunisie) \\ UR ECOTIDI (16ES10), Université virtuelle de Tunis) \\ ${ }^{4}$ (UMR EFTS/ Université de Toulouse-Jean Jaurès, France) \\ *Corresponding Author: Donia sghaier
}

\begin{abstract}
Résumé : Cet article s'intéresse aux pratiques ostensives de deux enseignants d'Education Physique et Sportive (EPS) dans l'activité natation en Tunisie. Son objectif est d'analyser les formes ostensives des enseignants d'EPS et de dégager leurs effets sur le rapport au savoir. La méthodologie de cette recherche est qualitative. Elle s'inscrit dans le champ des interactions en didactique clinique de l'EPS et sur l'analyse clinique des pratiques enseignantes des enseignants expérimentés et débutants afin d'entrevoir "le cas par cas » et le sujet singulier (Terrisse, 1999). Le recueil et l'analyse des données s'inscrivent dans une temporalité construite en un seul temps de l'action professorale : l'épreuve (Terrisse, 2000) tout en s'appuyant sur l'échelle des ostensions des savoirs (Robert, 2012; Salin, 2002). Les résultats mettent en évidence que la forme d'ostension utilisée par l'enseignant d'EPS influe sur le rapport au savoir en natation.
\end{abstract}

Mots clés - Ostensions, enseignants d'EPS, rapport au savoir, didactique clinique, natation.

\section{Introduction}

De nombreuses recherches en didactique des Mathématiques ont vu le jour autour de l'ostension, mais peu de recherches (Carnus, 2008 ; Robert, 2012 ; Carnus et Robert, 2013) se sont penchées sur l'ostension dans le cadre de la didactique de l'EPS. Cet article se propose alors d'étudier l'ostension « combinée » en natation comme un geste professionnel (Sghaier, Ben Jomâa, Mami \& Bouassida, 2016) qui met en avant le rapport au savoir de l'enseignant d'EPS.

En EPS, quelques travaux (Zimmerman 1976 ; Pujade-Renaud, 1977 ; Vigarello \& Vivès 1986 ; GalPetitfaux, 2000) soulignent le rôle du verbal puisqu'il est le support principal de l'enseignement. Cependant, tant d'auteurs (Quintillan, 1992 ; Kohler, 1998) soutiennent dans leurs études l'action du professeur, notamment dans les activités physiques et sportives (APS) où les relations entre enseignant et élèves impliquent le corps. Dans ce contexte, les situations d'enseignement, dans lesquelles l'enseignant présente plus ou moins directement le savoir aux élèves, sont qualifiées de pratiques «ostensives» (Salin, 2002). Elles représentent «des pratiques de communication d'un savoir, dans lesquelles l'enseignant fournit tous les éléments constitutifs de la notion visée » (Ratsimba-Rajohn, 1977).

A l'origine, le terme «ostension » appartient plutôt au vocabulaire liturgique (en anglais comme en français). Ce terme liturgique signifie alors des gestes qui "présentent aux fidèles » un objet du culte (croix, cierge, icône) ou une sainte espèce (reliques dans un reliquaire, Saint Sacrement). Par la suite, l'expression anglaise "ostensive action» a été traduite en terme d' "ostension» pour désigner que des légendes pouvaient être jouées et mises en actes, autant que racontées et mises en paroles. C'est pourquoi les spécialistes de la communication ainsi que les didacticiens des mathématiques se sont intéressés à ce phénomène d'ostension. Parmi ces didacticiens, nous citons Brousseau (1996) qui a étudié l'ostension et ses formes qui se combinent, de la plus "directe » à la plus «déguisée », en fonction du but recherché et de la stratégie de l'enseignant. Ce moyen, qui s'apparente à un "geste professionnel», nous paraît rendre compte d'une articulation chère à Brousseau : l'articulation « possible-nécessaire» (Brousseau, Op. cit).

Historiquement, Ratsimba-Rajohn (1977) a été le premier didacticien à identifier sous le nom d'« introduction ostensive » tout un ensemble de procédés didactiques qu'il considère comme irréductibles aux introductions par les définitions quel que soit le type. L'introduction ostensive des objets Mathématiques par ce dernier auteur (Ratsimba-Rajohn, Op. cit) a conduit Bautier (1988) à proposer une nouvelle définition du terme ostension : «L'ostension (...) dans une situation d'enseignement permet à l'élève de percevoir certains objets et l'illusion de porter une connaissance intellectuelle éventuellement générale et précise». Dans une telle 
approche, «l'ostension» se fait d'un cas particulier résolu, où l'enseignant donne «tous les éléments et relations constitutifs de la notion visée » (Ratsimba-Rajohn, Op. cit).

Pour Salin et Berthelot (1992), l'ostension est la résultante de la conception empiriste de la formation des connaissances. Ces deux didacticiens de Mathématique ont critiqué la forme d'ostension assumée pour les limites en géométrie. Ils ont considéré que cette ostension «assumée » a offert sa place à d'autres formes d'enseignement, telles qu'une forme d'ostension «déguisée » qui prédomine cependant la pratique en mathématiques des "activités ». C'est pourquoi, Salin (1999) a recensé les conclusions de nombreux travaux sur l'ostension. En 2002, Matheron et Salin constatent la persistance des pratiques ostensives et avancent que «l'ostension capture les autres procédés didactiques ».

Dans le même contexte, Frégona (1995) définit l'ostension comme « une réponse aux contraintes de la relation didactique ». Pour lui, l'ostension est une connaissance à la portée des enseignants. Elle permet avec certaines limites le contrôle de la relation didactique d'une manière stable et pragmatiquement efficace.

En général, une définition est par essence une ostension : « la désignation d'un objet pour lui faire jouer un rôle nouveau. Ce sont donc des ostensions dont les ostensifs sont construits pour les besoins d'une situation nouvelle » (Brousseau, 1996).

Ainsi, en tant qu'acte professionnel, Brousseau (Op. cit.) souligne que la combinaison entre les ostensions est un moyen qui s'apparente à un «geste professionnel ». D'ailleurs, cette « combinaison » paraît chère à cet auteur via "l'articulation possible-nécessaire ». Dans le même ordre d'idées, Berdot, BlanchardLaville et Dos Santos (1997) définissent l'ostension (du moins dans ses formes) comme « révélatrices de la distance de l'enseignant au savoir et comme identifiable par l'analyse et l'étude des différents gestes de direction d'étude de l'enseignant dans sa gestion des différentes ruptures de contrat ». Autrement dit, c'est la façon réelle pour l'enseignant de se distancier et de gérer plus ou moins ses savoirs en relation avec ceux proposés par les élèves.

Dès lors, nous pensons à travers ce phénomène que l'enseignant a le choix de plusieurs formes d'ostensions pour enseigner et que la combinaison de ces différentes formes ostensives pourrait être révélatrice du rapport au savoir, soit sa propre façon d'appréhender et de transmettre le savoir.

\subsection{L'ostension comme objet d'étude}

\section{Cadre conceptuel et notionnel}

Selon Sarrazy (2007), étant largement débattue et contre-versée, l'ostension est souvent présentée comme le symptôme d'une idéologie pédagogique dont l'empirisme constituerait la source. C'est le cas de Fregona (1995) pour qui «l'idéologie empiriste procure des solutions faciles à mettre en œuvre dans les décisions didactiques ». C'est aussi à ce même titre que l'ostension fut vivement condamnée par Aebli (1966) dans sa critique du sensualisme-empirisme qui inspira la didactique du même nom.

Considérée comme solution pour réduire les contraintes effectives de la relation didactique, RatsimbaRajohn (1992) la place lui aussi sous le joug d'une conception de la connaissance et la considère " comme seulement un système d'affirmations : règles, procédures, algorithmes, méthodes, procédés, définitions, etc. ». Dans le cas d'une activité enseignante en EPS, cette "conception » permet de pratiquer un transfert de la connaissance particulièrement utile comme un système d'affirmations à mémoriser et/ou à reproduire corporellement le savoir.

Selon Brousseau (1995), il est facile et efficace dans la vie courante de montrer un objet ou un animal en disant «voilà (vois là) une chaise » ou «voilà un chat» pour faire identifier un type d'objet, une espèce d'animal ou même une personne, en tant qu'élément générique d'une classe et en s'appuyant sur un répertoire de connaissance « universel» avec un jeu de variables laissées implicites. Dans ce cas, Brousseau (Op. cit.) parle de «communication de connaissance, ou plutôt de reconnaissance, [qui] ne passe pas par l'explicitation sous forme d'un savoir ». Pour décrire cette forme de communication, Brousseau utilise le mot « ostension » dérivant du terme latin « ostendere », qui signifie montrer, qui nous a par ailleurs donné les mots ostensible et ostentation, ainsi que l'ostensoir.

\subsection{Les pratiques ostensives en EPS}

Peu de recherches se sont penchées sur les pratiques ostensives en EPS. Seule la recherche de Robert (2012) a porté sur les pratiques ostensives non verbales en gymnastique. L'objectif de cet auteur était de dégager les convergences et les divergences relatives à l'activité d'enseignement de deux enseignants d'EPS à propos des pratiques ostensives dans un cours de gymnastique.

Pour Carnus et Robert (2013), ces pratiques ostensives sont des méthodes pour transmettre et/ou communiquer les enjeux de savoir. En ce qui concerne l'enseignant d'EPS, les actions ostensives, que ce soit verbales ou physiques, peuvent avoir une importance capitale en fonction du lieu, de l'espace, du moment et de l'activité enseignée. Plus spécifiquement, dans la discipline enseignée natation, si la nature des interactions varie entre professeur et élèves, il faut alors varier les procédures de communication et particulièrement les formes 
ostensives. Ces variations ostensives peuvent être le plus souvent tributaires de l'histoire singulière, de l'expérience et du vécu des sujets enseignants. C'est ce que nous tentons de mettre en évidence dans l'actuelle recherche à partir de la focale " effet de l'expertise sportive sur la pratique ostensive en enseignement». Dès lors, l'enseignant d'EPS se trouve, d'une part, face à plusieurs obstacles telles que les contraintes didactiques externes et notamment celles liées à la relation didactique et/ou à l'institution didactique. D'autre part, il est heurté à des contraintes liées à la logique interne et à la complexité motrice de la pratique de la natation.

Face à cette multiplicité d'obstacles difficiles à prévoir, l'un des rôles de l'enseignant consiste à gérer comme, il le peut, cette diversité d'obstacles. En effet, de sa nécessité de maintenir la relation didactique avec ses élèves (Sensevy, Mercier \& Schubauer-Léoni, 2000), il ne peut les laisser dans une situation d'échec permanent.

Alors, au travers des interactions didactiques, l'enseignant doit guider ses interventions en fonction des différents obstacles rencontrés par les élèves. Ensuite, il choisit le principal obstacle pour intervenir progressivement du facile au difficile. Mais, il peut aussi intervenir de différentes manières et surtout utiliser les formes d'ostensions ciblées et adaptées.

Face aux obstacles de la relation didactique, relatifs à la transmission des savoirs et à la proxémie, l'enseignant devient le seul responsable de l'émergence des savoirs. En effet, il tente de mobiliser des pensées réflexives, voire même stratégiques, pour intervenir. En conséquence, la seule solution qu'il peut faire c'est « donner à voir » du savoir au travers des procédures ostensives (Salin, 2002).

De manière corolaire, c'est l'expérience de l'enseignant qui lui permet d'achever à des solutions pendant sa séance. Ainsi, les pratiques ostensives sont considérées comme des méthodes et stratégies singulières que chaque enseignant met en œuvre pour intervenir et réguler la relation didactique. Pour cela, cette étude se propose d'analyser les pratiques ostensives des enseignants d'EPS en natation comme révélatrices de l'action professorale du rapport au savoir.

\subsection{Les formes ostensives}

En didactique des mathématiques, plusieurs formes d'ostensions ont fait l'objet d'étude de certains travaux. Parmi ces formes, nous citons l'«ostension assumée » (Salin \& Berthelot, 1992) pour laquelle le maître montre à l'élève ce qui est à voir. Nous citons également la forme « déguisée », apparue dans leur thèse (Salin \& Berthelot, Op. cit.) sous l'influence du constructivisme. Dans cette deuxième forme, c'est le maitre qui fait croire à l'élève qu'il découvre le savoir sur les figures soumises à son observation. Ainsi, ces auteurs soulignent que cette influence n'aurait pu avoir que des conséquences "de surface » générant alors une «forme déguisée d'ostension».

Par ailleurs, la première forme d'ostension mise en avant en EPS demeure la «monstration » du savoir tant considérée comme l'un des modes d'ostension permettant de communiquer par la démonstration gestuelle et/ou corporelle dite aussi ostension physique directe et/ou indirecte (manipulation du corps de l'élève qui démontre). Il s'agit de communiquer des informations relatives à un savoir en jeu (Bandura, 1986). Ainsi, la démonstration est liée au terme générique d'ostension. Cette démonstration, qu'elle soit silencieuse ou accompagnée d'explications verbales, est une procédure de guidage définie par Bandura (Op. cit.) dans le cadre de la théorie sociocognitive de l'apprentissage. Selon cet auteur, les savoirs et savoir-faire peuvent être acquis à partir de l'observation d'autrui. Dans le domaine des habiletés motrices, Carroll et Bandura (1985) ont utilisé ce modèle et ont prouvé la liaison entre les informations de guidage selon les différentes étapes de l'apprentissage. En effet, lors de la première phase d'apprentissage, les démonstrations du modèle demeurent souvent efficaces.

Dans la même lignée des idées, les travaux de Lafont (1994), Laugier (1995) et Cadopi (1995) ont mis en évidence l'efficacité de la modélisation et de la démonstration explicitée lors des phases précoces des acquisitions. D'après Serre (1984), dans le cas des activités morpho-cinématiques telles que la danse et la natation synchronisée.

Il s'avère alors que la démonstration est bel et bien efficace pour les habiletés à production de forme avec une pression temporelle limitée. Mais il reste que l'image du geste à réaliser, n'est pas une garantie de réussite, dans la mesure où elle occulte tout le travail de tâtonnement et de recherche créatrice propice aux apprentissages. Dès lors, la variation et la combinaison des modes d'ostension utilisés par l'enseignant permettent de spécifier le rapport au savoir de l'enseignant et de révéler son action professorale.

Par ailleurs, plusieurs formes d'ostensions et/ou de procédures ostensives peuvent coexister dans l'agir de l'enseignant lors de l'épreuve d'enseignement de natation : l'ostension directe, verbale et/ou gestuelle. Nous développerons cette partie ultérieurement. Buznic (2009) recense trois formes d'ostensions et/ou d'expositions des savoirs : l'explicitation par le discours, la manipulation du corps de l'élève et la démonstration par l'intermédiaire du corps de l'enseignant. Ainsi, à partir des travaux de recherches préalablement effectués en EPS (Buznic, 2004 ; Guirat, 2005 ; Léal, 2006 ; Ben Jomâa, 2009), nous pouvons identifier plusieurs niveaux d'ostension de savoirs en EPS : 
- L'ostension directe physique : le savoir est démontré physiquement par l'enseignant. Lorsqu'elle est réalisée par l'enseignant, cette forme ostensive peut revêtir deux significations. En montrant aux élèves qu'il maîtrise les savoirs en jeu, il «appuie son autorité didactique » (Buznic, 2009) mais aussi, d'un point de vue plus intime, il «s'affirme d'un plaisir narcissique en montrant aux autres qu'il sait» (Buznic, Op. cit.). La posture du «sujet supposé savoir» (Lacan, 1964) rend compte de cette recherche de plaisir narcissique et de volonté d'asseoir son autorité didactique.

- L'ostension verbale directe et l'ostension verbale particulière : Dans le premier cas, le savoir est énoncé, présenté et/ou expliqué à l'élève de façon directe. Les éléments cognitifs constitutifs du savoir sont sous la responsabilité de l'élève. Carnus (2008) parle d'approche langagière verbale. Dans le second cas, l'enseignant d'EPS ne maîtrise pas les savoirs moteurs où ne peut plus « démontrer ». Il peut avoir recours à une forme d'ostension verbale particulière. Pour ce faire, il utilise ses connaissances scientifiques (issues de la biomécanique, de la psychologie cognitive, ect.).

- L'ostension physique privée : Cette manipulation peut être totale ou partielle. Nous qualifions ainsi l'ostension physique privée de totale et de partielle. En fonction du degré d'intervention de l'enseignant, les éléments moteurs et cognitifs constitutifs du savoir peuvent être en partie sous la responsabilité de l'élève. Dans une étude portant sur une enseignante débutante, Buznic (2004) montre qu'indifféremment de son niveau d'expertise dans l'activité enseignée, l'enseignante a recours à ce type d'ostension. Il y voit la difficulté pour un professeur sans expérience de proposer des situations dans lesquelles le savoir est entièrement dévolu aux élèves et qui relèvent nécessairement d'une certaine complexité de conception et de gestion des contenus d'enseignement. En effet, « il lui est ainsi difficile de mettre en jeu les gestes de direction d'étude complexes que sous-tendent les pratiques d'enseignement non ostensives » (Buznic, 2004).

- L'ostension déguisée où le savoir est montré par l'intermédiaire d'une mise en avant des objets du milieu « détenteurs du savoir » au travers de la justification de certaines conditions du milieu ou des buts de la situation, ou par l'intermédiaire d'un questionnement avec l'élève sur ces objets pertinents.

- L'ostension par aménagement du milieu est à rapprocher de l'ostension déguisée, quand celle-ci se rapproche d'un aménagement excessif du milieu. L'enseignant a la possibilité de moduler la contrainte des mouvements du corps vers une gestuelle choisie à travers l'aménagement du dispositif matériel. En effet, « des élèves peuvent faire partie de ce dispositif avec comme rôle de guider, d'aider, de manipuler l'apprenti dans ses réalisations motrices. Pour distinguer cette forme d'ostension de l'ostension déguisée, que nous réservons au moment d'interactions verbales, nous parlerons d'ostensions aménagée » (Léal, 2006).

- Pas d'ostension : le savoir naît par un questionnement sur l'activité de l'élève. Dans ce cas, tous les éléments constitutifs du savoir sont sous la responsabilité de l'élève. En conséquence, le processus d'ostension renvoie au rapport qu'entretient un enseignant avec le savoir dans ses interactions didactiques avec les élèves pour en connaître les caractéristiques. Dès lors, à travers le geste, la démonstration du savoir permet de donner aux élèves « une image » plus précise et plus rapide du résultat final à atteindre par les élèves.

De manière corolaire, l'intérêt de notre étude consiste d'abord à déterminer les différentes formes ostensives utilisées par les enseignants d'EPS susceptibles d'orienter leurs interventions ostensives vers des obstacles spécifiques. Ensuite, nous envisageons d'analyser les pratiques ostensives observées chez les enseignants révélatrices du rapport au savoir.

\section{Problématique et question de recherche}

Dans le cadre de l'enseignement de l'EPS, l'activité natation se fait souvent dans des conditions difficiles, où la communication est perturbée et la proxémie entre l'enseignant et les élèves est instable. Cependant, l'apport d'informations se fait à condition que l'élève arrête son action, ce qui interrompt l'activité dans l'eau et contraint l'évolution de l'apprentissage. Dès lors, la seule mise en mot des contenus par l'enseignant peut sembler insuffisante et incomprise dans le milieu aquatique.

Dans ces conditions complexes, comment les élèves pourraient-ils comprendre ce que dit l'enseignant et comment l'enseignant de natation pourrait-il alors transmettre le savoir ? De ce fait, la problématique de recherche s'intéresse aux formes ostensives en natation, tout en étudiant l'effet des pratiques ostensives des enseignants d'EPS sur le rapport au savoir.

Cet article sur les pratiques ostensives en natation se veut exploratoire et originale du moins en milieu universitaire tunisien. Se situant dans cette problématique, nous nous interrogeons sur l'impact de ses formes. Ainsi, à travers cette étude, nous tentons de répondre aux questions suivantes :

Est-ce que les formes d'ostensions sont suffisantes pour une communication efficace en milieu aquatique ? Ou bien, faut-il combiner entre ces différentes formes?

Est-ce que l'ostension combinée pourrait révéler le rapport au savoir en natation?

En s'inspirant des travaux de Brousseau (1996), qui souligne que la combinaison entre les ostensions est un moyen qui s'apparente à un «geste professionnel », notre objectif est de montrer que la variation et la 
combinaison entre les formes ostensives par l'enseignant n'est pas un phénomène spontané et hasardeux. Dès lors, nous postulons que cette combinaison est l'objet d'une véritable expertise dans l'APS enseignée et qu'elle est révélatrice du rapport au savoir.

\section{Méthode et outil}

Nous sommes partis de l'hypothèse suivante: la combinaison entre les formes d'ostensions par l'enseignant est révélatrice du rapport au savoir. Pour traiter cette hypothèse, nous nous sommes d'abord inscrits dans le champ de la didactique clinique de l'EPS, par la suite nous avons réalisé une étude de cas auprès des deux enseignants d'EPS et de leurs étudiants inscris en $1^{\text {ème }}$ année LFEP ${ }^{1}$ à l'ISSEP ${ }^{2}$ de Tunis. La finalité de l'étude est descriptive et compréhensive et n'a pas pour objectif la modélisation et la généralisation.

\subsection{La population d'étude}

Dans le cadre de notre recherche, une pré-observation a été effectuée pour s'imprégner des spécificités de la classe et habituer l'enseignant et les élèves à la présence du chercheur et de la caméra. L'expérimentation a été réalisée, au sein de l'université de la Manouba à la Piscine de Bardo. Deux classes d'étudiants de la $1^{\text {ère }}$ Année LFEP ont été retenues pour l'expérimentation de notre protocole. Chaque classe a été soumise à des séances d'apprentissage de la nage Crawl, niveau "Nageur». Les étudiants ont été encadrés par deux enseignants spécialistes de natation disposant de degré d'expertise et d'expériences professionnelles différentes. Nous appelons les deux enseignants associés : l'expert (E1) et la novice (E2).

\subsection{Protocole d'observation}

Nous avons observé, pour chacun des deux enseignants associés, une séance au milieu du cycle portant sur la nage Crawl. Cette observation, au cœur de la phase d'apprentissage dans le cycle, nous garantit une densité didactique. Selon Marsenach et Mérand (1987), les premières séances consacrées à diverses évaluations ne sont que peu riches en contenus d'enseignement. La première observation s'est déroulée avec l'enseignant expert et la deuxième observation s'est déroulée avec l'enseignante novice. Chaque séance a duré environ 45 minutes.

\subsection{Méthodologie didactique clinique}

La didactique prend en compte, l'ensemble des méthodes, techniques et procédés pour l'enseignement. Elle met principalement l'accent sur les moyens d'enseigner, sur le comment faire. Ainsi, Terrisse (1998) justifie l'intérêt de l'approche clinique en didactique de l'EPS, par la prise en compte de la singularité du sujet dans le processus d'enseignement-apprentissage, et poursuit en disant que : ce qui fait son originalité, c'est l'articulation entre les concepts de la didactique qui tentent de cerner au plus près le savoir transmis, et la clinique qui intègre la contingence de cette transmission et le fait que ces savoirs sont transmis par des sujets « divisés » (Terrisse, 2009).

Face aux contraintes du milieu aquatique, l'enseignant doit intervenir de différentes manières, comme il peut «donner à voir» du savoir au travers des procédures ostensives diverses (Salin, 2002). Dès lors, l'enseignant d'EPS utilise les formes ostensives entendues comme des stratégies particulières, des modalités de mises en œuvre, des réponses adaptées, le plus souvent, aux contingences du milieu, qui peuvent à des moments particuliers de l'épreuve améliorer son rapport au savoir.

Sachant que cette recherche s'inscrit dans l'orientation didactique clinique de l'EPS, notre méthodologie privilège l'étude de cas et se veut qualitative. Cette méthodologie se base sur l'étude « au cas par cas, au un par un » (Terrisse, 1999) afin d'appréhender les sujets dans leur singularité et dans leur complexité. Cette singularité est prise en compte en didactique clinique par une attention particulière à l'histoire personnelle de chaque enseignant au travers de l'étude de son déjà-là (Carnus, 2003), c'est-à-dire la prise en compte a priori et a posteriori de « ce qui singularise chaque enseignant et donc ses pratiques d'un point de vue épistémologique et didactique » (Buznic, 2009).

\subsection{Recueil des données}

La méthode de recueil et d'analyse de données s'inscrit dans une temporalité construite en trois temps distincts et croisés de l'action professorale : le déjà-là, l'épreuve et l'après-coup (Terrisse, 2000) (tableau 1). Cependant, dans le cadre de cet article, nous nous sommes limités au deuxième temps, celui de l'épreuve. Les pratiques ostensives ont été décrites et catégorisées à partir de l'enregistrement audio et vidéo des séances et des données d'entretiens semi directifs et/ou verbatim (a priori, ante et post séance et d'après-coup).

\footnotetext{
${ }^{1}$ Licence Fondamentale en Education Physique.

${ }^{2}$ Institut Supérieure du Sport et de l'Education Physique de Tunis, Tunisie.

DOI: $10.9790 / 7388-0704023644 \quad$ www.iosrjournals.org $\quad 40 \mid$ Page
}


Tableau 1. Le recueil temporel des données (Terrisse, 2008).

\begin{tabular}{|c|c|c|}
\hline Déjà-là & Epreuve & Après-coup \\
\hline $\begin{array}{c}\text { Conception de l'APS Conception } \\
\text { de son enseignement. }\end{array}$ & $\begin{array}{c}\text { Observation in situ : confrontation } \\
\text { du savoir prévu à la classe. }\end{array}$ & $\begin{array}{c}\text { Retour sur les séances d'EPS } \\
\text { nouvelle formulation. }\end{array}$ \\
\hline
\end{tabular}

Le traitement des données recueillies s'organise également selon ces trois temps distincts de la méthodologie didactique clinique. Il s'agit dans cet article d'étudier seulement le deuxième temps, celui de l'épreuve, qui correspond à la confrontation du sujet enseignant à l'épreuve de transmission du savoir. Pendant l'épreuve, l'enregistrement audio et vidéo des séances est complété par un entretien ante séance et un entretien post séance à chaud. Le recueil et l'analyse des données s'appuient ainsi sur l'échelle des ostensions des savoirs (Salin, 2002 ; Robert, 2012).

Nous avons utilisé le logiciel SportsCode V10.3 sur un MacBook Pro 15 avec 2,66 Ghz Intel Core i5 CPU, 8GB RAM et $7200 \mathrm{rpm}$ hard drive avec Mac OS X El Captain version 10.11 pour découper l'enregistrement vidéo et indexer chaque extrait afin d'obtenir un graphique qui rend compte de ces découpages en fonction de leur durée.

Dans la fenêtre de codification de SportsCode, notre choix de boutons code et de labels nous permet de nous concentrer sur des indicateurs de la séance qui nous seront utiles à analyser. Notre fenêtre de codification/codage peut être simple ou complexe selon notre mode de fonctionnement et peut évoluer au cours de la séance.

Une fois que la vidéo est associée à la Timeline, la codification/codage peut aussi être faite manuellement et nous pouvons ainsi visualiser les séquences déjà codées.

\section{Résultats}

Les résultats obtenus pour chaque étude de cas sont illustrés dans le graphique 1 selon l'épreuve (Terrisse, 2000) et/ou le temps effectif de l'action professorale.

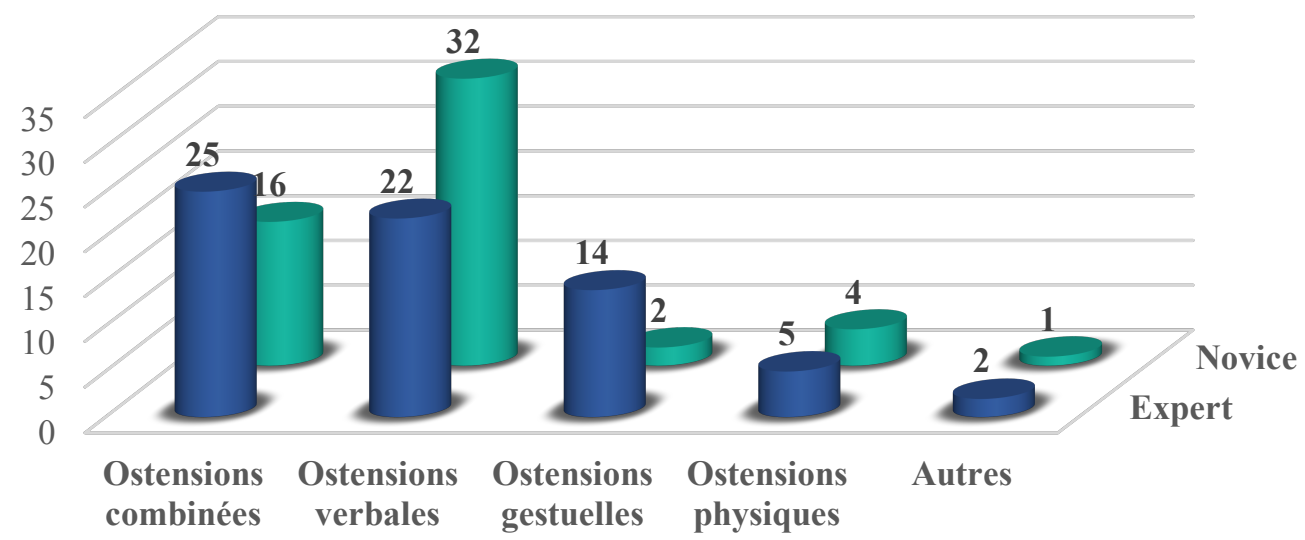

Graphique 1. Les formes d'ostensions des savoirs de E1 et E2.

\section{Discussion}

A travers l'échelle des ostensions des savoirs (Salin, 2002 ; Robert, 2012), l'analyse du graphique 1 de l'épreuve nous a permis d'illustrer des divergences entre les pratiques ostensives des deux enseignants. Pendant l'épreuve de 1'E1, nous avons dénombré 25 ostensions combinées. En effet, E1 privilège le geste. Le verbal complète le geste et est utilisé en deuxième lieu. Il se tient en distance personnelle de conversation, d'où il est un peu plus à distance des étudiants. Ce dernier utilise préférentiellement dans l'interaction enseignant/étudiant souvent une partie combinée. Ainsi, nous avons identifié 14 ostensions de type gestuel symbolique dont deux oratoires et rythmiques. Nous avons enregistré également 5 ostensions physiques privées sous formes de démonstrations partielles de geste. Toutefois, le nombre dominant des ostensions combinées, gestuelles symboliques et physiques privées enregistré dans le site de l'E1 montre qu'il transmet le plus souvent une expérience personnelle et/ou une expertise corporelle, faite de «son vécu personnel» de spécialiste et «du ressenti corporel » (Ben Jomâa \& Terrisse, 2014) dont le corps fait le « seul lieu d'aventure » (Vigarello, 1982).

A travers ces différentes formes ostensives, l'enseignant expert adapte ses interventions en fonction des capacités et des intentions de ses apprenants afin d'améliorer son rapport au savoir. Ainsi, il régule l'apprentissage de ses étudiants tout en « personnalisant » par son propre corps les « traits pertinents » du savoir à produire, ce qui est en mesure de «co-construire» la référence en classe (Schubauer-Léoni, 2008). Cette 
référence représente, par le biais de son expression corporelle, une forme de «contrat didactique ». Il s'agit notamment d'un point de repère à respecter, gage de réussite pour les étudiants. Cette référence alimente une mémoire didactique ou «mémoire de classe » (Brousseau \& Centeno, 1991) en devenant peu à peu une histoire didactique au fil d'une leçon, d'une séquence ou d'une année ce qui révèle une expérience corporelle professionnelle. Pour cela, ces pratiques ostensives combinées et à dominante gestuelle sont des images de savoir et des messages véhiculés par le corps de l'enseignant qui révèlent son rapport "personnel» et/ou "privé » au savoir et témoigne de son expertise sportive en natation.

A l'instar, lors de son épreuve d'enseignement, l'E2 a fait principalement recours aux ostensions verbales avec 32 ostensions, dont 14 verbales particulières et 18 directes. Pour les ostensions combinées, nous n'en avons dénombré que 9 par rapport aussi à 2 ostensions gestuelles symboliques lors de la séance de l'E2. Pour elle, c'est le verbal qui est premier et le geste complète son propos. Elle se tient très proche de ses étudiants pour mieux les garder sous contrôle et elle n'apprécie pas de démontrer physiquement, ce qui se traduit dans son activité professorale par son « impossible à supporter » : s'exprimer corporellement.

Cependant, le nombre réduit des ostensions combinées et gestuelles enregistré dans le site de l'E2 montre qu'elle énonce et explique souvent le savoir verbalement. En effet, elle a tendance à adopter une stratégie verbale et/ou discursive d'enseignement et de passer par le canal cognitif de l'étudiant puisqu'elle a recours soit à la forme d'ostension verbale directe, soit à la forme particulière. Ces deux formes ostensives verbales demeurent insuffisantes quand il s'agit de l'apprentissage technique. Les travaux de Vigarello et de Vivès (1983) ont déjà montré que le discours technique nécessite des détours par des phases gestuelles. Cette interprétation est également soutenue par Marsenach et Mérand (1987) qui considèrent que la gestualité fait partie des pratiques ostensives de l'enseignant d'EPS.

A travers ces formes ostensives verbales auxquelles l'E2 a eu manifestement et couramment recours pour communiquer, nous constaterons qu'elle ne maitrise pas les savoirs moteurs et qu'elle ne veut pas « démontrer » physiquement et ceci révèle son rapport «distant» et/ou " officiel» au savoir scolaire faute d'expérience professionnelle, notamment, dans l'enseignement de l'APS natation.

\section{Conclusion}

A travers l'échelle des ostensions des savoirs (Salin, 2002; Robert, 2012), les résultats mettent en évidence que les formes ostensives utilisées par l'enseignant expert sont variées. Alors que les formes ostensives de la novice sont limitées. De ce fait, l'utilisation de plusieurs formes d'ostensions à différents moments de la même situation peut nous permettre de favoriser l'implication des étudiants dans les apprentissages. Dans ce sens, nous pouvons en référer à Beillerot (1996), pour qui le rapport au savoir est un «processus créateur de savoir pour un sujet-auteur, nécessaire pour agir et pour penser ». Autrement dit, le savoir est reconstruit et reformulé par le sujet lui-même, en fonction de sa propre expérience corporelle (ici sportive) et professionnelle.

Par la suite, l'enseignant expert adapte ses interventions en fonction des capacités et des intentions de ses apprenants afin d'améliorer son rapport au savoir. Ainsi, le nombre des ostensions combinées met en évidence son expérience corporelle et professionnelle qui font penser à la fois à l'« expression corporelle, langage du silence » (Pujade-Renaud, 1977) et au « corps parlant» (Jorro, 2004).

Contrairement à l'enseignant expert, les pratiques ostensives de l'enseignante novice sont limitées et peu variées. Elles se déploient souvent sous formes d'ostensions verbales qui sont ponctuelles, brèves et superficielles. Dès lors, la parole et les mots s'avèrent impuissants à exprimer la complexité des situations et l'émergence de plusieurs éléments simultanément au moment où le geste permet de concrétiser ce qu'il y a à voir et à comprendre. En conséquence, l'absence de l'image de savoir à donner (la référence) à ces apprenants ne permet pas de construire et/ou d'établir le contrat didactique et ne créé pas des rapports au savoir avec ces étudiants pour qu'ils puissent tirer profit de sa manière de transmettre les savoirs. Par la suite, l'ostension prend une dimension prédominante dans la relation qui unit l'enseignant au savoir.

En effet, Berdot, Blanchard-Laville et Dos Santos (1997) définissent l'ostension (du moins dans ses formes) comme « révélatrices de la distance de l'enseignant au savoir et comme identifiable par l'analyse et l'étude des différents gestes de direction d'étude de l'enseignant dans sa gestion des différentes ruptures de contrat ». Autrement dit, c'est la façon réelle pour l'enseignant de se distancier et de gérer plus ou moins ses savoirs en relation avec ceux proposés par les élèves.

Enfin, l'ostension, qui constitue la focale de cet article, demeure intéressante à étudier dans toutes ses formes puisqu'elle permet de dévoiler l'implicite et les enjeux de l'acte d'enseignement. A travers ces formes d'ostensions, l'enseignant fait ses propres choix didactique et combine entre ces différentes formes qui sont révélatrice du rapport que l'enseignant entretient avec le savoir. Dès lors, le rapport au savoir diffère de l'enseignant expert à celui du novice. Par la suite, il est influencé non seulement par son action didactique, mais aussi par sa compétence professionnelle.

En conséquence, le choix de l'intervention efficace et la variation des gestes d'études s'inscrivent dans la complexité de la relation didactique entre l'enseignant, l'élève et le savoir. 
La perspective de cette recherche consiste à penser cette combinaison comme une stratégie didactique susceptible d'aider les enseignants d'EPS, et particulièrement les enseignants de natation, à optimiser leur activité d'enseignement.

\section{Références}

[1]. Aebli, H. (1966). Didactique psychologique : application à la didactique de la psychologie de Jean Piaget, $4^{\text {ème }}$ ed., Neuchâtel : Delachaux et Niestlé, $163 \mathrm{p}$.

[2]. Bandura, A. (1986). L'Apprentissage social. Mardaga.

[3]. Bautier, T. (1988). Une modélisation didactique des activités d'enseignement des premières propriétés de la symétrie orthogonale, Séminaire de didactique des mathématiques et de l'informatique, LSD-IMAG, Institut Fourier, Université J. Fourier, Grenoble, année 86-88.

[4]. Beillerot, J. (1996). Pour une clinique du rapport au savoir. Paris : L’Harmattan.

[5]. Ben Jomâa, H. \& Terrisse, A. (2014). L'effet d'un "déjà-là » sur la pratique d'un enseignant: le point de vue de la didactique clinique de l'EPS. Revue Recherches en Education, $n^{\circ} 20$, p.116-126.

[6]. Ben Jomaa, H. (2009). L'expertise dans l'enseignement de l'EPS : analyse didactique clinique de quatre professeurs. Thèse de doctorat en cotutelle. Université Paul Sabatier. Toulouse III, Université de la Manouba (Tunisie).

[7]. Berdot, P. ; Blanchard-Laville, C. \& Camara Dos Santos, M. (1997). La construction de l'espace psychique dans la classe. In Blanchard Laville, C. (dir.), Variations sur une leçon de mathématiques. Paris : L’Harmattan.

[8]. Berthelot, R. \& Salin, M.-H. (1992). L'enseignement dans l'espace et de la géométrie dans l'enseignement obligatoire. Thèse de l’Université Bordeaux I, Talence.

[9]. Brousseau, G. \& Centeno, J. (1991). Rôle de la mémoire didactique de l'enseignant. Éditions, la pensée sauvage. Revue Recherche en Didactique des Mathématiques, vol n¹1, pp. 167-210.

[10]. Brousseau, G. (1996). Cours 2 : Les stratégies de l'enseignant et les phénomènes typiques de l'activité didactique. In R. Noirfalise et M.-J. Perrin-Gionan (éds), Actes de la VIIIe École d'Été de didactique des mathématiques. Clermont-Ferrand : IREM, p. 16-30.

[11]. Buznic, P. (2004). De l'expérience à l'enseignement. Une étude de cas didactique d'une enseignante d'EPS débutante. Mémoire de DEA en Sciences de l'éducation, sous la direction d'A. Terrisse, UFR Espaces et Sociétés, Université Toulouse Le Mirail, document non publié. UTM.

[12]. Buznic, P. (2009). La transmission du savoir expérientiel. Etudes de cas et analyses comparatives en didactique clinique de l'EPS. Thèse non publiée, Université Paul Sabatier, Toulouse.

[13]. Cadopi, M. (1995). Représentations cognitives et performance dans les actions morpho-cinétiques. In H. Ripoll et al (Coord.), Psychologie du sport. Questions actuelles (p. 237-248). Paris : Editions Revue EPS.

[14]. Carnus, M.-F. (2003). Analyse didactique du processus décisionnel de l'enseignant d'EPS en gymnastique : une étude de cas croisés. In : C Amade-Escot (éd) Didactique de l'éducation physique, état des recherches, 195-224, Paris : Revue EPS.

[15]. Carnus, M.-F. (2008). Analyse didactique clinique de pratiques d'enseignants expérimentés et débutants. Une étude de cas croisée sur l'usage de la notion de «gainage » en cours d'éducation physique et sportive (E.P.S). In Carnus, M-F. Garcia-Debanc, C. Terrisse, A. (dir.). Analyse des pratiques des enseignants débutants, approches didactiques, (éd) La Pensée Sauvage, pp 213-232.

[16]. Bandura. A (1985). Role of timing of visual monitoring and motor rehearsal in observational learning of actions patterns, Journal of Motor Behavior, vol. 17

[17]. Fregona, D. (1995). Les figures planes comme milieu dans l'enseignement de la géométrie: interactions, contrats et transpositions didactiques. Thèse de l'Université Bordeaux I, Bordeaux.

[18]. Gal-Petitfaux, N. (2000). Typicalité dans la signification et l'organisation de l'intervention des professeurs d'EPS en situation d'enseignement de la Natation : le cas des situations de nage en file indienne. Thèse non publiée de doctorat en STAPS, Université Montpellier.

[19]. Guirat, N. (2005). L'étude sur l'impact de l'expérience sur l'enseignement de l'EPS : le cas d'un enseignant. Mémoire de Mastère en S.T.A.P.S. Université Paul Sabatier (Toulouse) et de la Manouba (Tunisie) Guyot, Y. (1970). Relations professeur-étudiants : étude expérimentale des rapports professeur élèves au travers des attitudes à l'égard de l'espace pédagogique Paris, C.D.U.-SEDES, p.76.

[20]. Jorro, A. (2004). Le corps parlant de l'enseignant. Actes du $9^{\text {ème }}$ colloque de l'AIRDF, Québec, 26-28 Août.

[21]. Kohler, M. (1998). La démonstration partielle des enseignants d'EPS en gymnastique sportive : une technique corporelle pour une meilleure représentation du geste. Dossier EPS $N^{\circ} 39$. Paris : Éditions revue EPS, pp. 84-88.

[22]. Lacan, L. (1964). Sujet supposé savoir. En ligne http ://lexique-de-lacan.blogspot.com/2010/08/sujet-suppose-savoir.html.

[23]. Lafont, L. (1994). Modalités sociales d'acquisition d'habiletés motrices complexes. Thèse en psychologie non publiée. Université René Descartes Paris V, Paris.

[24]. Laugier, C. (1995). Apprentissage par observation en danse : rôle des processus représentatifs dans la reproduction de mouvements. Thèse en STAPS non publiée. Université de Montpellier I, Montpellier.

[25]. Léal, Y. (2006). L'analyse didactique clinique de pratiques ostensives : une étude de cas croisés en EPS au collège et à l'école primaire. Mémoire de Master recherche $2^{\text {ime }}$ année.

[26]. Marsenach, J. \& Merand, R. (1987). L'évaluation formative dans les collèges, Paris, INRP.

[27]. Matheron, Y. \& Salin, M.-H. (2002). Les pratiques ostensives comme travail de construction d'une mémoire officielle de la classe dans l'action enseignante. In Revue Française de Pédagogie, $n^{\circ}$ 141, octobre novembre décembre 2002, pp. 57-66.

[28]. Pujade-Renaud, C. (1977). Expression corporelle langage du silence, Editions E.S.F., Science de l'Education, $4^{e}$ édition.

[29]. Quintillan, G. (1992). Enseignement sportif et communication non verbale. La gestualité spécifique de l'entraîneur. Thèse de doctorat en Sciences de l'éducation, non publiée, Université de Paris V.

[30]. Ratsimba-rajohn, H. (1977). Etude de l'introduction ostensive d'un objet mathématique. Mémoire de DEA. Université Bordeaux 1.

[31]. Ratsimba-rajohn, H. (1992). Contribution à l'étude de la hiérarchie implicative. Application à l'analyse de la gestion didactique des phénomènes d'ostension et de contradictions. Thèse de l'Université Rennes I, Rennes.

[32]. Robert, M. (2012). L'ostension non verbale en EPS : Analyse didactique clinique des pratiques gestuelles de deux enseignants aux profils contrastés lors de l'enseignement de la rotation avant. Mémoire en Master 2 recherche Éducation, Formation, Travail et Savoirs, UTM.

[33]. Robert, M. \& Carnus, M-F. (2013). Les formes ostensives non verbales en gymnastique constitutives de la signature professionnelle de deux enseignants d'EPS. In M-F. Carnus et A. Terrisse (Dir). Didactique clinique de l'EPS. Le sujet enseignant en question. Revue EPS (eds.), 81-92.

[34]. Salin, M.-H. (1999). Pratiques ostensives des enseignants et contraintes de la relation didactique. In G. Lemoyne et F. Conne (éds), 
Le cognitif en didactique des mathématiques. Montréal : Les Presses de l'Université de Montréal, p.327-352.

[35]. Salin, M.-H. (2002). Les pratiques ostensives dans l'enseignement des mathématiques comme objet d'analyse du travail du professeur. In O. Venturini, C. Amade-Escot et A. Terrisse (éds). Etude des pratiques effectives: l'approche des didactiques. Grenoble : La Pensée Sauvage, pp.71-81

[36]. Sarrazy, B. (2007). Ostension et dévolution dans l'enseignement des mathématiques : anthropologie wittgensteinienne et théorie des situations didactiques. Education et Didactique, 1, 3, p. 31-46.

[37]. Schubauer-Leoni, M.L. (2008). Co-construire des savoirs : les métiers de l'intervention dans les APSA. Actes du colloque ARIS Besançon 9-12 mai 2006 au PUF-C. (psychologue sociale de formation).

[38]. Sensevy, G. ; Mercier, A. \& Schubauer-Léoni, M.-L., (2000). Vers un modèle de l'action didactique du professeur à propos de la course à 20. Recherches en didactique des mathématiques, 20/3.

[39]. Serre, J.C. (1984). La danse parmi les autres formes de la motricité, La recherche en danse, 3, 135-156.

[40]. Sghaier, D. ; Ben Jomaa, H. ; Mami, M. \& Bouassida, A. (2016). The combination of the physical ostension to the verbal ostension in swimming revealing of professorial action. Creative education, 7, pp. 500-505.

[41]. Terrisse, A. (1998). Transposition didactique et prise en compte du sujet: perspectives de recherche en éducation physique et sportive. In Amade-Escot, C.; Barrué, J.P.; Bos, J.-C.; Dufor, F; Dugrand, M.; et Terrisse, A. : Recherches en EPS, bilans et perspectives. Paris : Éditions Revue EPS, pp 81-90.

[42]. Terrisse, A. (1999). La question du rapport au savoir dans le processus d'enseignement-apprentissage : le point de vue clinique, Carrefour de l'éducation, 7, pp. 62-87.

[43]. Terrisse, A. (2000). Epistémologie de la recherche clinique en sports de combat. Recherches en sports de combat et en arts martiaux : état des lieux et perspectives. Collection Recherche et Formation. Paris : Editions Revue EPS.

[44]. Terrisse, A. (2008). Le sujet en didactique clinique de l'EPS : Conditions et conséquences pour la recherche. eJRIEPS, 15, pp. 179197. Besançon : IUFM de Franche-Comté. (Version électronique).

[45]. Terrisse, A. (2009). La didactique clinique en EPS. Origine, cadre théorique et recherches empiriques. In A. Terrisse et M.-F. Carnus, Didactique clinique de l'EPS, quels enjeux de savoirs? (p. 13-31). Bruxelles : De Boeck.

[46]. Vigarello, G. (1982). Les vertiges de l'intime. In Le corps entre illusions et savoirs. Revue Esprit, 2. Paris : Editions revue Esprit.

[47]. Vigarello, G. \& Vivès, J. (1983). Technique corporelle et discours technique, Revue EPS, $n^{\circ} 184$ pp.40-47.

[48]. Vigarello, G. \& Vivès, J. (1986). Discours de l'entraîneur et technique corporelle. Revue EPS, 200-201, 146-153.

[49]. Zimmermann, D. (1976). Les voies non verbales de la relation pédagogique. Edition ESF science de l'éducation. 